\title{
O fenol como agente neurolítico no tratamento da espasticidade
} focal e multifocal

\section{Phenol as a neurolithic agent in the treatment of focal and multifocal spasticity}

Dayrin Vanessa Tarazona Carvajal ${ }^{1}$, Natália de Oliveira Silva² ${ }^{2}$ Lucas Soiti Maeda ${ }^{3}$, (D Marcelo Riberto ${ }^{4}$

\section{RESUMO}

A síndrome piramidal ocorre nas lesões do sistema nervoso central que afetam as vias corticoespinhais e são definidas pela tríade de fraqueza muscular, aumento dos reflexos miotáticos e espasticidade, que é definida pelo aumento involuntário da resistência ao movimento passivo cuja intensidade varia com a velocidade do movimento. Quando a espasticidade interfere na funcionalidade do paciente, dificultando o movimento ativo, causando dor ou dificultando a prestação de cuidados por terceiros, há necessidade de iniciar seu tratamento. Para o tratamento da espasticidade generalizada ou de grandes porções do corpo, a intervenção medicamentosa ocorre por via oral ou intratecal, conforme os recursos disponíveis, mas efeitos colaterais de intensidade variável e indesejáveis podem ocorrer, especialmente o comprometimento da atenção ou da consciência, que prejudicam o processo de reabilitação. $O$ tratamento focal da espasticidade utiliza a toxina botulínica ou os bloqueios nervosos com fenol ou álcool. Este artigo revisa a literatura sobre as técnicas mais adequadas para realizar a neurólise com fenol. Os bloqueios neuromusculares com fenol são um tratamento efetivo, de ação imediata, baixo custo, duração prolongada e de poucos efeitos adversos quando são respeitados os cuidados regulares de aplicação.

Palavras-chave: Fenol, Espasticidade Muscular, Bloqueio Nervoso, Terapêutica

${ }^{1}$ Residente de Medicina Física e Reabilitação, Faculdade de Medicina da Universidade de São Paulo - FMUSP

2 Médica Neurologista, Mestranda em Neurologia, Faculdade de Medicina de Ribeirão Preto - FMRP-USP

${ }^{3}$ Médico Neurologista, Hospital das Clínicas da Faculdade de Medicina de Ribeirão Preto FMRP-USP

4 Professor Associado, Departamento de Biomecânica, Medicina e Reabilitação do Aparelho Locomotor, Faculdade de Medicina de Ribeirão Preto - FMRP-USP

\section{Correspondência}

Marcelo Riberto

E-mail: mriberto@usp.br

Submetido: 14 Julho 2019.

Aceito: 23 Julho 2019.

\section{Como Citar}

Carvajal DVT, Silva NO, Maeda LS, Riberto M. O fenol como agente neurolítico no tratamento da espasticidade focal e multifocal. Acta Fisiatr. 2019;26(1):59-65.

DOI: 10.11606/issn.2317-0190.v26i1a163003

\section{ABSTRACT}

The pyramidal syndrome occurs in central nervous system injuries that affect the corticospinal pathways and are defined by the triad of muscular weakness, increased myotatic reflexes and spasticity, which is defined by the involuntary increase in resistance to passive movement that varies in intensity according to the velocity of joint movement. When spasticity interferes with the patient's functioning, making it difficult to actively move, causing pain, or making it difficult to receive care from others, treatment must be stated. For the treatment of generalized spasticity or for large portions of the body, drug intervention may be used either orally or intrathecally, depending on available resources, but undesirable and variable intensity side effects may occur, especially impairment of attention or awareness, which further delays the rehabilitation process. Focal treatment of spasticity utilizes botulinum toxin or nerve blocks with phenol or alcohol. This article reviews the literature on the most suitable techniques for performing phenol neurolysis. Neuromuscular blockade with phenol is an effective treatment, with immediate action, low cost, prolonged duration and few adverse effects when the regular care of application is respected.

Keywords: Phenol, Muscle Spasticity, Nerve Block, Therapeutics

\section{cc) (i) (2)}




\section{INTRODUÇÃO}

A espasticidade é uma desordem do controle motor resultante de uma lesão no neurônio motor superior que se apresenta como ativação muscular involuntária. Diversas doenças comuns em nosso meio podem provocar lesões no neurônio motor superior e consequentemente, espasticidade. Assim o manejo da espasticidade é importante na prática clínica diária de fisiatras, neurologistas, fisioterapeutas, terapeutas ocupacionais e todos profissionais de saúde envolvidos na reabilitação de paciente neurológicos.

Clinicamente, a espasticidade caracteriza-se pelo aumento do tônus muscular dependente da velocidade e é acompanhada por outros sinais da síndrome do neurônio motor superior como clônus, espasmos ou contrações simultâneas de grupos antagonistas.

O sinal clínico característico ao exame físico é o "sinal do canivete", no qual o segmento corporal que é movimentado passivamente com velocidade angular veloz apresenta resistência maior no início do arco de movimento e redução brusca da resistência no final. ${ }^{1}$

A espasticidade pode complicar o quadro clínico original da doença de base e impactar negativamente a funcionalidade do paciente uma vez que pode reduzir a amplitude de movimentos, ocasionar dor, atrapalhar o sono e reduzir a sua capacidade funcional.

Em casos mais graves, os pacientes evoluem com encurtamento muscular, retrações tendíneas, deformidades ortopédicas e necessidade de abordagens cirúrgicas, o que aumenta a morbidade e os custos de tratamento.

Entretanto, nem todos pacientes requerem tratamento para espasticidade. Alguns pacientes utilizam o aumento do tônus para desenvolver mecanismos compensatórios e facilitar algumas atividades funcionais, como postura ortostática e auxílio nas transferências, o que torna necessário avaliar a real necessidade do seu tratamento. Por isso, avaliação deve ser individualizada, pautada no objetivo funcional pactuado e priorizado pelo paciente e cuidadores. $^{2}$

O tratamento da espasticidade pode ser multimodal e combinar diversas técnicas: medicamentos orais, bloqueios químicos (toxina botulínica e/ou fenol), bomba intratecal de baclofeno, cirurgias para correção de deformidades. É indispensável associar medidas nãofarmacológicas, como fisioterapia motora, terapia ocupacional e uso de órteses. ${ }^{3}$

A escolha da modalidade do tratamento depende das características da espasticidade, especialmente se é generalizada, focal ou multifocal. Na espasticidade generalizada, o uso de medicamentos com efeito de relaxamento muscular é frequente; enquanto na espasticidade focal ou multifocal, os bloqueios neurolíticos, com fenol, álcool ou toxina botulínica, constituem a principal opção de tratamento.

Este artigo destaca o bloqueio neurolítico com fenol como opção eficaz e de baixo custo para o tratamento da espasticidade focal e multifocal, especialmente naqueles pacientes com grande quantidade músculos acometidos, nos quais o tratamento com toxina botulínica pode tornar-se bastante oneroso.

Aborda, ainda, as características químicas, histórico de utilização, mecanismo de ação, dose e técnicas dos principais sítios de aplicação. ${ }^{4}$

As indicações de tratamento da espasticidade estão resumidas no (Quadro 1).5

\section{Propriedades químicas do fenol}

O fenol é um composto químico que contém ácido carbólico, ácido fênico, ácido fenílico, hidróxido de fenila, hidroxibenzeno e oxibenzeno. 6

Trata-se de uma substância muito solúvel em solventes orgânicos, tais como álcool ou glicerol. Normalmente, utilizam-se soluções aquosas de fenol entre $3,0 \%$ até $7,0 \%$ ( $25 \%$ de fenol em $60 \%$ de solução de glicerina diluída em água estéril na concentração de $5 \%$ ). ${ }^{7}$
Quadro 1. Indicações para tratamento da espasticidade 5

\section{Indicação}

Exemplos

Aprimoramento da movimentação ativa

Alivio dos sintomas e questões subjetivas e movimentos involuntários

Redução da necessidade de cuidados

Aprimorar respostas futuras à reabilitação
Melhorar a postura
Aumento da velocidade e precisão do movimento; aprimoramento dos parâmetros lineares da marcha ou de segurança, ou na propulsão de cadeira de rodas. Melhor agilidade para as transferências. Melhora na destreza ao alcançar objeto. Melhora

Melhora na dor e da sensação de rigidez associada à espasticidade.

Melhora da imagem corporal.

Previne a instalação e agravamento de contraturas musculares e deformidades.

Adequação do equilíbrio muscular periarticular. Redução de espasmos musculares.

Reduz a necessidade de ajuda para higiene e cuidados pessoais, ajuste de órteses e adaptações, mobilização e posicionamento.

Melhor adesão aos exercícios durante a terapia e às recomendações domiciliares. Prevenção de contraturas e aprimoramento da imagem corporal. nas condições para relação sexual.

\section{História do uso clínico do fenol}

As primeiras descrições históricas do uso de fenol no âmbito da saúde datam dos anos de 1860, quando foi usado como agente antisséptico durante procedimentos cirúrgicos. Posteriormente, no início do século $X X$, foram realizadas as primeiras aplicações em nervos para tratamento de neuralgias. Apenas em 1919 o fenol foi usado pela primeira vez para o tratamento da espasticidade, por meio da aplicação muscular direta; em seguida, vieram as publicações referentes ao uso intratecal e, por último, as neurólises de nervos periféricos. ${ }^{8}$

O uso da via intratecal para bloqueio das raízes anteriores dos nervos espinhais foi desencorajada ao longo dos anos devido às significativas complicações relatadas: ${ }^{9}$ danos das raízes nervosas, paralisia motora, infarto medular, perda da sensibilidade, meningite, parestesia dolorosa e óbito. ${ }^{10} \mathrm{Em}$ vista disso, em 1966, descreveu-se uma via de administração alternativa, com bloqueios de pontos motores, reportando melhora da espasticidade em 39 pacientes a longo prazo. ${ }^{11}$

Os bloqueios com fenol foram muito utilizados para o tratamento da espasticidade nas décadas de 1970 e 1980; porém, comparado com a toxina botulínica tipo A (TBA), o fenol tem sido menos utilizado nas últimas décadas, apesar de ser efetivo como agente químico desnervante, ter início de ação imediata, baixo custo e, muitas vezes, uma duração equiparável ao efeito da TBA. ${ }^{10,12}$

A perda de popularidade do bloqueio neuroquímico por meio deste fármaco pode ser decorrente do desconhecimento por parte de muitos médicos sobre as técnicas para a sua administração, o temor associado aos seus efeitos adversos e a maior simplicidade de aplicação da TBA. Na atualidade, com o conhecimento de que a disestesia é muito rara quando se bloqueia nervos com predomínio de fibras motoras, ressurgiu o tratamento com fenol em forma de procedimento misto associado a toxina em casos de espasticidade multifocal, com o propósito de tratar mais músculos em uma única sessão sem exceder as doses de ambos agentes. ${ }^{13}$

O uso combinado de fenol como uma alternativa possível de tratamento da espasticidade foi descrito por 16 de 49 médicos brasileiros, dos quais $73 \%$ relataram seu uso em 1 a 5 pacientes por semana. O uso isolado do bloqueio fenólico foi descrito por $55 \%$ desses médicos, apesar de $45 \%$ deles preferirem o uso combinado com a TBA. 
O bloqueio nervoso mais comumente realizado é do ramo anterior do nervo obturatório ( $67,2 \%$ dos pacientes), ramo motor do nervo ciático (35\%), nervo musculocutâneo $(20,6 \%)$, nervo femoral (18\%), ramos motores do nervo tibial (15\%) e pontos motores dos músculos em $10 \%{ }^{14}$

\section{Mecanismo de ação do fenol}

O mecanismo de ação do fenol parece ser dual. Por um lado, o fármaco atua como um anestésico local sobre as fibras gama e por outro, produz axoniotomese química, ou seja, desorganiza a estrutura dos axônios, apesar de ainda manter os tubos endoneurais. ${ }^{9}$ Ocorre proteólise, comprometimento do componente lipídico da membrana celular e separação da bainha de mielina dos axônios, com consequente interrupção dos sinais eferentes das células hiperexcitáveis do corno anterior da medula, por meio de uma necrose induzida. ${ }^{7,13,15}$

O resultado é a interrupção nervosa do arco reflexo e, consequentemente, diminuição do tônus muscular. ${ }^{9}$ Os efeitos da neurólise química com fenol não são permanentes, pois pode ocorrer reinervação funcional num período de meses ou anos. ${ }^{9,16,17}$

\section{Indicação do uso do fenol}

A indicação de neurólise química é a existência de espasticidade com comprometimento funcional ou doloroso, que prejudique a qualidade do ato motor ou resulte em deformidades articulares. ${ }^{18}$

0 tratamento da espasticidade modifica significativamente 0 processo de reabilitação já que qualquer que seja a sua topografia, a presença de dor intensa à mobilização articular impede a reabilitação efetiva. Em pacientes com espasticidade focal e multifocal, alguns estudos indicam que o bloqueio com fenol teria um efeito superior na lesão cerebral traumática e nas lesões medulares que nos pacientes com acidente cerebrovascular devido ao maior tempo de recuperação. ${ }^{9}$

\section{Dose}

A quantidade aplicada varia de $0,6 \mathrm{ml}$ a $3,0 \mathrm{ml}$ por ponto. ${ }^{19,20}$ Geralmente, inicia-se com doses baixas, havendo aumentos posteriores para obtenção de efeitos mais adequados. No entanto, deve-se individualizar o paciente e o nervo a ser tratado. A dose letal estimada varia entre $8,5 \mathrm{~g}-15,0 \mathrm{~g}$ e não se recomenda administrar mais de $1 \mathrm{~g}$ em 24 horas, ou seja, $20,0 \mathrm{ml}$ de fenol a $5 \% .{ }^{17,21,22}$

\section{Efeitos adversos}

A acidez do fenol pode causar efeito inflamatório local, além disso, possui baixo potencial de difusão tecidual, portanto, deve ser injetado o mais próximo possível ao nervo alvo para obter melhores resultados e menos efeitos adversos locais. ${ }^{7}$

Caso o fenol seja injetado próximo a fibras sensitivas pode causar disestesia e dor neuropática, o que ocorre em 2-32\% dos procedimentos em pacientes adultos. ${ }^{23,24}$

Tal efeito adverso pode durar até 4 meses e afetar a relação dos segmentos corpóreos, implicando em modificação da postura e podendo comprometer os ganhos obtidos pelo procedimento nas atividades da vida diária dos pacientes.

Por isso, a neurólise por fenol para tratamento da espasticidade deve ser realizada em nervos com função exclusiva ou predominantemente motora; logo, a realização de procedimentos guiados pode diminuir o risco de acometimento das fibras sensitivas.

Outros efeitos adversos incluem edema, eritema cutâneo, trombose venosa profunda e infecção local. ${ }^{17,22,25}$ Imediatamente após a aplicação, os pacientes podem apresentar cefaleia, sensação de embriaguez, hálito alcoólico, náuseas ou vômito. Estes efeitos colaterais costumam remitir espontaneamente em menos de uma hora. ${ }^{7,26}$

\section{Materiais e equipamentos}

Para ter maior efeito de redução da espasticidade e dor, bem como reduzir a ocorrência de efeitos adversos, o bloqueio neuromuscular com fenol exige que a aplicação do fármaco seja orientada com maior precisão possível junto ao nervo-alvo. Isso exige que o procedimento seja guiado por um recurso de imagem, usualmente ultrassonografia, ou por eletroestimulação, que serão o foco das orientações de materiais a seguir:

- Agulha para anestesia plexular revestida de teflon, com calibre 22 a 28, cujo comprimento deve ser baseado no local do procedimento (1,2 a 3,0 polegadas);

- Seringa de $5 \mathrm{ml}$ com conector de extensão para agulha - extensão necessária para melhor estabilização de agulha durante aspiração e injeção do medicamento;

- Eletroestimulador com estímulo elétrico de 1 milissegundo e pulso de onda quadrada;

- Eletrodo gel para conexão do polo positivo do eletroestimulador;

- Gaze e álcool 70\% para higienização do local do procedimento;

- Fenol a 5\% para injeção.

\section{Técnica}

A seguir estão descritas as técnicas de neurólise de quatro nervos periféricos que são amplamente abordados: A alça dos peitorais, o nervo musculocutâneo, o nervo obturatório e os ramos motores do nervo tibial. O nervo mediano, por ter grande componente sensitivo, não será abordado nesta revisão, já que o risco de disestesia secundária é significativa. ${ }^{21}$

Apesar da recomendação habitual na literatura mais recente de técnicas que utilizem a ultrassonografia e eletroestimulação simultaneamente para melhor localização do nervo a ser abordado, uma vez que oferece uma visualização direta do nervo e outras estruturas vasculares a serem evitadas.

Essa combinação também promove a redução do volume requerido de fenol, devido a maior facilidade de localização do nervo. ${ }^{16,27,28}$ No entanto, tendo em vista a necessidade do equipamento e de habilidades no manejo do aparelho de ultrassom, essa localização combinada foge da intenção desta revisão, que é descrever uma abordagem mais prática e acessivel.

Além isso, a abordagem dos nervos guiadas por marcos anatômicos associada a utilização de eletroestimulação se mostrou tão efetiva quanto à abordagem combinada. ${ }^{28}$

Após a higienização do local de punção com álcool 70\%, conectase o eletroestimulador à agulha de anestesia de plexo e ao eletrodo é colado ao corpo do paciente, preferencialmente junto a proeminências ósseas mais próximas.

A seringa com o conteúdo de fenol $5 \%$ deve ser conectada ao extensor e à agulha, preenchendo todo o trajeto com o medicamento. Insere-se a agulha no marco anatômico em que se localiza o nervo e ajusta-se o eletroestimulador com o objetivo de alcançar o máximo de contração dos músculos desejados com o mínimo de corrente.

Esse processo pode exigir que a corrente aplicada inicialmente seja maior, ou seja, por volta de 3,0 a 4,0 mA, o que provoca estímulo direto de músculos.

A mobilização progressiva da agulha e redução progressiva da corrente elétrica permite identificar a posição da agulha com a melhor relação da intensidade da contração muscular com menor estímulo (< $1 \mathrm{~mA}$ ), indicando maior proximidade com o nervo-alvo.

Deve-se injetar o fenol no volume de 0,6 a $3,0 \mathrm{ml}$ lentamente no ponto de melhor resposta ao estímulo elétrico, até que a contração seja eliminada, ${ }^{7}$ indicando a efetividade da neurólise. ${ }^{17,18,29}$ 
Porém, deve-se verificar a resposta e, se for o caso, injetar uma dose maior, tendo em vista que o efeito do fenol é dose-dependente. ${ }^{7}$

Nem todos os nervos devem ser abordados com esse agente em pacientes com sensibilidade íntegra, preferindo-se aqueles com componente motor exclusivo ou com pouco componente sensitivo. Em pacientes com lesão medular completa, por exemplo, a liberdade para neurólise sobre nervos com grande componente sensorial é maior, uma vez que não haverá ocorrência de dor neuropática.

Quando o fenol é administrado, nota-se uma diminuição praticamente instantânea do tônus. O fenol tem um longo período de ação que vai desde 6 meses a 1 ano, podendo chegar até um ano e meio. ${ }^{21}$ Esse tempo varia com a concentração do fármaco, o volume utilizado e a técnica usada. . $^{27,30}$

\section{Localizações anatômicas}

\section{Nervo obturatório}

O ramo anterior do nervo obturatório inerva os músculos adutor longo, adutor curto e grácil, enquanto o ramo posterior inerva os músculos adutor magno e obturador externo. Este é, provavelmente, o nervo mais comumente abordado na neurólise química, apresentando como resultado redução da espasticidade de adutores. $^{31}$

Neste artigo, descreveremos três técnicas diferentes abordando seus pontos positivos e negativos. $\mathrm{O}$ uso de ultrassonografia para guiar o procedimento implica em índices de sucesso de $90 \%$ a $100 \%$, porém não foram realizados estudos comparativos com outras técnicas de localização. 32

\section{Abordagem clássica simplificada ou púbica}

Inicialmente descrito por Labat em 1922, esta técnica foi simplificada por Park em 1967,33 os quais utilizavam apenas marcos anatômicos. Em 1984, Gasparich et al. ${ }^{34}$ incluiu a eletroestimulação à técnica para melhor localização do nervo obturatório.

Para este procedimento, o paciente é colocado na posição classicamente descrita de litotomia, porém a abordagem com o paciente em decúbito dorsal com coxa levemente abduzida e rodada externamente também foi descrita sem alteração da técnica. ${ }^{35}$

Insere-se a agulha a 2,0 cm lateral e $2,0 \mathrm{~cm}$ caudal do tubérculo púbico, com um pequeno desvio cefálico até que seja feito contato com ramo superior do osso púbico. Após, desliza-se a agulha para a margem inferior desse ramo ósseo e aprofunda-se pósterosuperiormente mais $2,0 \mathrm{~cm}$. Com a agulha nessa posição, liga-se o eletroestimulador para localização mais precisa do nervo obturatório.

Nesta técnica, tenta-se abordar o nervo antes de sua ramificação em anterior e posterior. No enfoque tradicional, realiza-se uma punção perto do tubérculo púbico, o que pode ocasionar dor ao contato com o osso e um risco maior de lesão de órgãos como a bexiga ou a vagina, ${ }^{19}$ por isso vem sendo estudadas outras técnicas para abordar os ramos anterior e posterior separadamente e distalmente à área inguinal.

\section{Abordagem inguinal}

Choque et al. ${ }^{35}$ propuseram uma técnica para localizar os ramos anterior e posterior separadamente.

Realizaram um teste prévio com cadáveres frescos e posteriormente realizaram os bloqueios em pacientes com o procedimento a seguir: com o paciente é colocado em posição supina e as coxas levemente abduzidas e rodadas externamente, procura-se o tendão do músculo adutor longo (o tendão palpável mais superficial) e o pulso da artéria femoral sobre a prega cutânea inguinal como pontos de referência.

Marca-se o ponto médio entre esses pontos sobre a prega inguinal, o qual corresponde ao centro de um sulco facilmente palpável entre a artéria e o músculo adutor longo. A agulha é inserida nesse ponto em um ângulo de $30^{\circ}$ na direção cefálica até observar as contrações do músculo adutor longo e do grácil, local provável do ramo anterior do nervo obturatório.

Após bloqueio, pode-se aprofundar ligeiramente a agulha, com discreto desvio lateral até ser observado contração do músculo adutor magno (provável local do ramo posterior do nervo obturatório) (Figura 1).

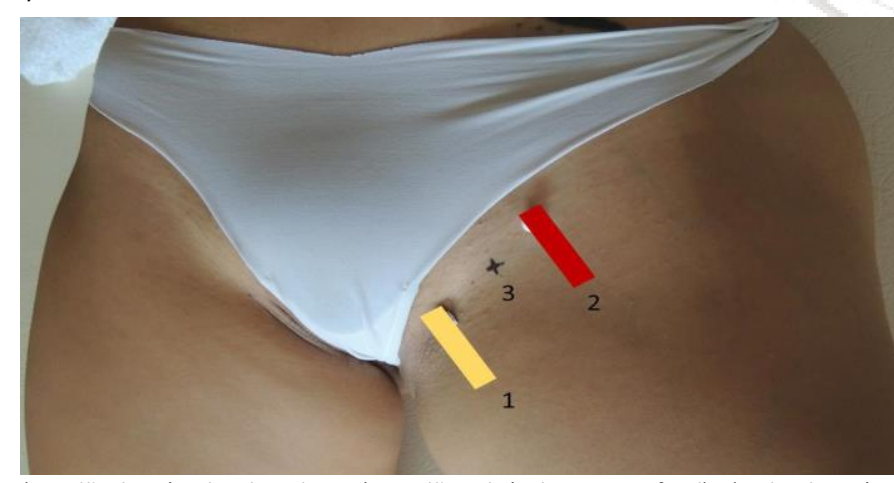

1) Tendão do músculo adutor longo (O tendão palpável mais superficial), 2) Pulso da artéria femoral, 3) Local onde é inserida a agulha em um ângulo de $30^{\circ}$ na direção cefálica

Figura 1. Localização do ramo anterior do nervo obturatório

Esta técnica é mais rápida e causa menor desconforto para o paciente em comparação com a técnica clássica, além de mesma se obter a mesma qualidade no bloqueio. ${ }^{35}$

\section{Abordagem interadutores}

A técnica descrita por Wassef em $1993^{36}$ consiste na abordagem interadutores do ramo anterior do nervo obturador e mostrou ser um acesso mais fácil e com melhor acurácia em comparação a técnica clássica, apresentando a mesma taxa de sucesso no bloqueio. $6,37,38$

Consiste na inserção da agulha a $1,0 \mathrm{~cm}$ abaixo do tendão do músculo adutor longo e $2,0 \mathrm{~cm}$ lateral ao arco púbico com o paciente em posição de litotomia (Figura 2). A agulha deve ser introduzida com uma inclinação ligeiramente posterior.

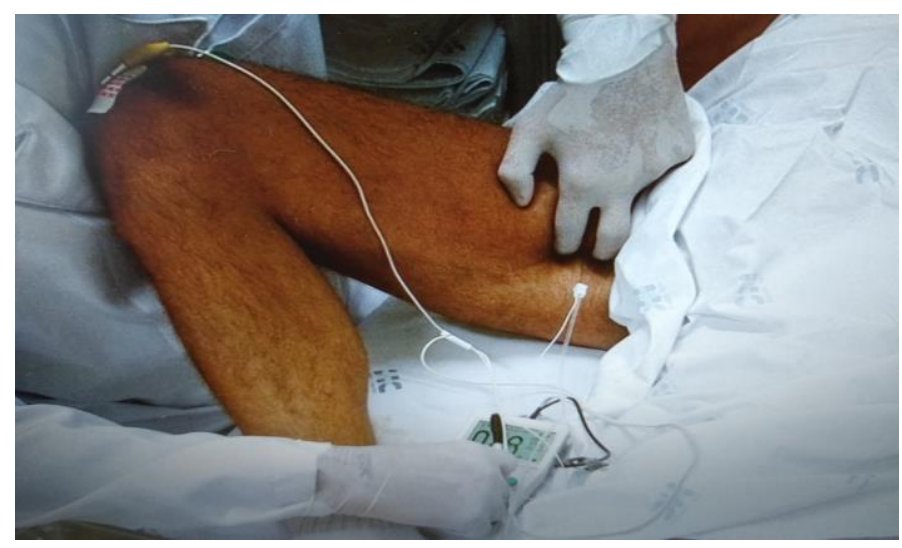

Figura 2. Posicionamento da agulha de eletroestimulação entre os músculos adutor longo e magno

\section{Nervo tibial}

É o segundo nervo mais comumente bloqueado, sendo utilizado para tratar a espasticidade dos músculos da panturrilha. ${ }^{6}$

O nervo tibial inerva os músculos gastrocnêmio, solear, poplíteo, extensor longo dos dedos, extensor longo do hálux, além de vários intrínsecos do pé.

O bloqueio deste nervo tem sido utilizado para tratar o pé equinovaro, o qual é principalmente causado pela espasticidade do 
solear, gastrocnêmio e tibial posterior ${ }^{29}$ ocasionando melhora no ortostatismo e na marcha, reduzindo a flexão plantar e o varismo do pé. ${ }^{39,40}$

No entanto, o tronco principal do nervo tibial não é um nervo exclusivamente motor, tendo em vista presença de fibras sensitivas que inervam a planta do pé, ${ }^{29}$ podendo ocorrer efeitos adversos de dor e disestesia, ${ }^{28}$ contudo uma série recente de pacientes tratados com essa abordagem apresentou dor neuropática em apenas 2,2\%. ${ }^{41}$

Em vista disso estudos recentes têm focado na localização dos ramos motores para maior seletividade e menos efeitos adversos. ${ }^{29,39}$

\section{Ramos motores do nervo tibial aos gastrocnêmios}

Esses ramos motores originam-se obliquamente do tronco do nervo tibial a uma distância média de 3,3+/-1,2 cm acima da linha horizontal da margem superior da cabeça da fíbula.

A agulha deve ser inserida no limite inferior destes ramos (localizada por eletroestimulação), 1,0 cm medial e lateral ao nervo tibial acima da linha horizontal formada pela margem superior da cabeça de fíbula, a uma profundidade de aproximadamente 1,0 a 3,0 $\mathrm{cm}^{39}$ (Figuras 3).

O nervo tibial pode ser localizado utilizando-se o eletroestimulador ou sua posição definida aproximadamente por uma linha que vai do tendão de Aquiles até o ponto médio da linha horizontal formada pela cabeça da fíbula.

\section{Ramo motor do nervo tibial para os músculos solear e tibial posterior}

Há poucos estudos de bloqueios seletivos destes ramos na literatura, possivelmente pela sua dificuldade de localização, tendo em vista serem muito pequenos, com não mais de $2,0 \mathrm{~mm}$ de diâmetro.

Deltombe et al. ${ }^{29}$ localizaram esses ramos mediante tomografia e definiram as coordenadas que posteriormente utilizaram para bloqueio com auxílio de estimulador elétrico em 12 pacientes com espasticidade de membro inferior.

O ramo motor ao músculo solear esteve localizado a 10,0 $\pm 5,0$ $\mathrm{mm}$ abaixo da linha horizontal que atravessa a cabeça da fíbula, a 17,0 $\pm 9,0 \mathrm{~mm}$ lateral a uma linha mediana na panturrilha que vai desde 0 ponto médio da fossa poplítea ao tendão de Aquiles e a 47,0 $\pm 4,0$ $\mathrm{mm}$ de profundidade (Figura 4).

O ramo motor do músculo tibial posterior foi localizado a $45,0 \pm$ 6,0 $\mathrm{mm}$ abaixo da cabeça da fíbula, 17,0 $\pm 8,0 \mathrm{~mm}$ lateral a uma linha mediana na panturrilha que vai desde o ponto médio da fossa poplítea ao tendão de Aquiles e 47,0 $\pm 4,0$ de profundidade (Figura 4).

\section{Alça dos peitorais}

A alça dos peitorais corresponde aos nervos peitoral medial e lateral, ambos, ramos do plexo braquial, ${ }^{18}$ os quais inervam os músculos peitoral maior e menor. A espasticidade do peitoral maior induz uma deformidade em adução e em rotação interna. Essa limitação na abdução do braço ocasiona dor regional ${ }^{18,42,43}$ e limitação para a higiene e a troca de roupas.

O ponto de inserção da agulha corresponde a união do terço médio ao terço externo de uma linha que une a parte superior da axila a articulação esternoclavicular ${ }^{18}$ (Figura 5), o que se aproxima razoavelmente da proposta do Özel et al. ${ }^{43}$ com abordagem direta aos nervos peitorais medial e lateral a partir de um estudo em cadáveres. ${ }^{31}$

Os resultados do bloqueio neurolítico mostram uma significante redução nas escalas de espasticidade e de dor. ${ }^{18}$

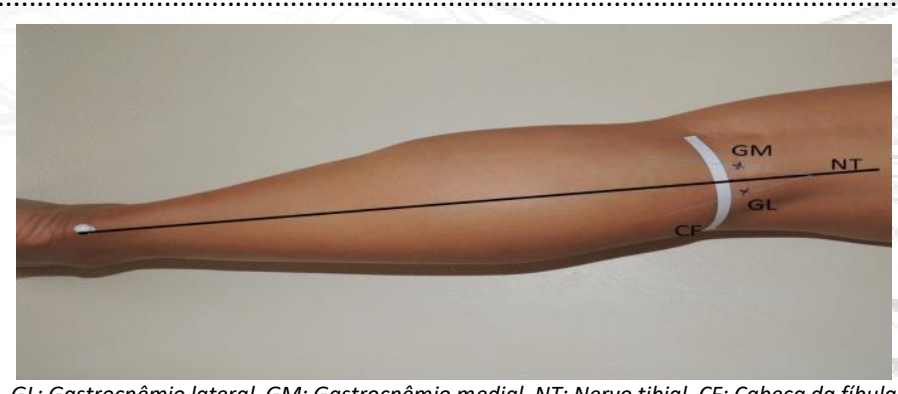

GL: Gastrocnêmio lateral, GM: Gastrocnêmio medial, NT: Nervo tibial, CF: Cabeça da fíbula

Figura 3. Localização dos ramos motores do Nervo tibial ao gastrocnêmio lateral e medial

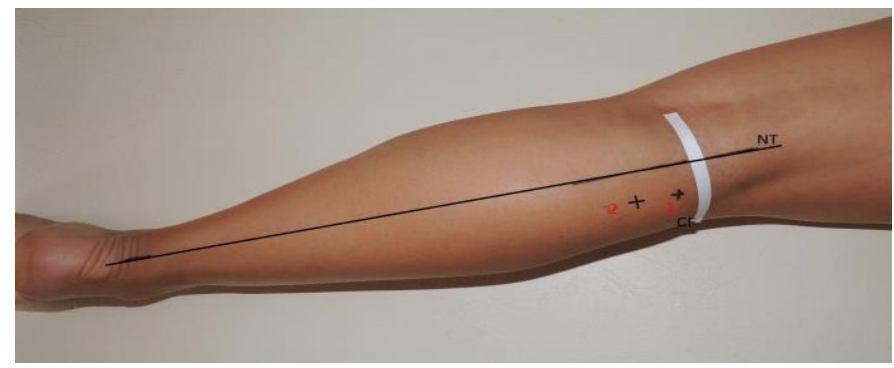

1) Localização cutânea do ramo motor do nervo tibial ao músculo sóleo, 2) Localização cutânea do ramo motor do nervo tibial ao músculo tibial posterior, NT: Nervo tibial, CF: Cabeça da fíbula

Figura 4. Ramo motor aos músculos sóleo e tibial posterior

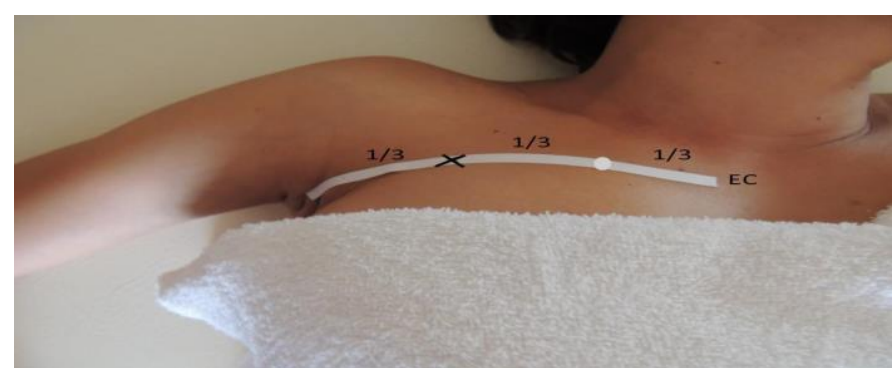

EC: Articulacao esternoclavicular

Figura 5. Alça dos peitorais, união do terço médio ao terço externo de uma linha que une a parte superior da axila a articulação esternoclavicular

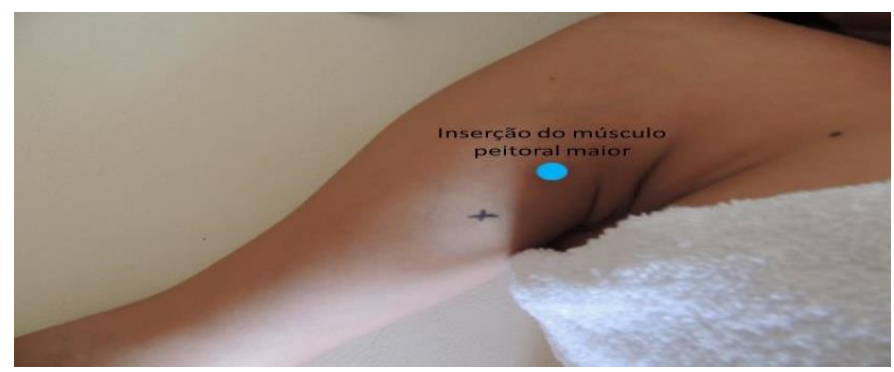

Figura 6. Nervo musculocutâneo a 2 polpas digitais distal a inserção do músculo peitoral maior, superior a artéria braquial

\section{Nervo musculocutâneo}

Responsável pela inervação dos três músculos anteriores do braço: o coracobraquial, o bíceps braquial e o braquial anterior, que são os grandes flexores do cotovelo. O nervo pode ser identificado a 2 polpas digitais distalmente à inserção do músculo peitoral maior na parte superior do braço. Palpa-se a artéria braquial e insere-se a agulha 1,0 $\mathrm{cm}$ anterior à artéria e dirigida anterolateralmente até que se produza a contração do bíceps ${ }^{22,44}$ (Figura 6). 
É recomendável estar atento ao padrão de contração produzido pela estimulação elétrica, pois o estímulo ao nervo mediano pode contrair os músculos flexores dos dedos e do carpo, produzindo algum grau de flexão do cotovelo, dada a origem de tais músculos no epicôndilo medial do úmero. Desta forma, tanto a estimulação do nervo musculocutâneo como do nervo mediano podem causar flexão do cotovelo ao ritmo dos pulsos do gerador elétrico. A estimulação do nervo musculocutâneo, porém, não resulta em flexão do punho ou dos dedos, o que serve para diferenciá-la da estimulação do nervo mediano.

O bloqueio inadvertido do nervo mediano pode ser muito problemático, uma vez que seu componente sensorial é muito pronunciado e as queixas de disestesia e dor podem prevalecer.

$\mathrm{Na}$ eventualidade de perceber a contração rítmica do punho e dedos frente à estimulação elétrica, o médico que realiza este procedimento deve tracionar a extremidade da agulha para uma posição mais superficial e apontá-la em direção anterior até obter a contração vigorosa dos flexores do cotovelo.

\section{CONCLUSÃO}

A neurólise com fenol pode ser uma ferramenta de escolha para tratamento de pacientes com espasticidade quando o uso da toxina botulínica apresentar limitações em relação a doses e custos. É um tratamento efetivo, de ação imediata, de baixo custo, efeito a longo prazo e poucos eventos adversos quando se bloqueiam nervos de predomínio motor.

$\mathrm{Na}$ atualidade, tem-se utilizado cada vez mais métodos de bloqueio de nervos guiados por ultrassonografia, ${ }^{38}$ porém, tendo em vista limitações técnicas e financeiras destes novos métodos, a utilização da anatomia de superfície para guiar a localização para bloqueios de nervos, associado à eletroestimulação, ainda se mostra uma técnica útil e eficaz.

\section{REFERÊNCIAS}

1. Lianza S, Musse $\mathrm{Cl}$, Bernardo W, Silvinato A, Simões R. Neurólise com fenol no tratamento da espasticidade. Med Reabil. 2015;34(3):79-81.

2. Mullins $D$, Winter $A$, Fini $N$, Dixon $C$, Aldous $S$, Palit $M$, et al. Frequency and characteristics of goal attainment following BoNTA injection for management of spasticity. Disabil Rehabil. 2016;38(19):1927-33.

DOI: https://doi.org/10.3109/09638288.2015.1107781

3. Laxe S. Which interventions are useful for managing muscle spasticity in individuals who sustained traumatic brain injury? - A Cochrane Review summary with commentary. NeuroRehabilitation 2019;44(1):157-9. DOI: https://doi.org/10.3233/NRE-189003

4. Escaldi S. Neurolysis: a brief review for a fading art. Phys Med Rehabil Clin N Am. 2018;29(3):519-27. DOI: https://doi.org/10.1016/j.pmr.2018.03.005

5. Ward $A B$. Spasticity treatment with botulinum toxins. J Neural Transm (Vienna). 2008;115(4):607-16. DOI: https://doi.org/10.1007/s00702-007-0833-2

6. Ghai A, Garg N, Hooda S, Gupta T. Spasticity - Pathogenesis, prevention and treatment strategies. Saudi J Anaesth. 2013;7(4):453-60.

7. Kolaski KL, Logan LR. Intrathecal baclofen in cerebral palsy: A decade of treatment outcomes. J Pediatr Rehabil Med. 2008;1(1):3-32.

8. Khalili AA, Harmel MH, Forster S, Benton JG. Management of spasticity by selective peripheral nerve block with dilute phenol solutions in clinical rehabilitation. Arch Phys Med Rehabil. 1964;45:513-9.

9. Jozefczyk PB. The management of focal spasticity. Clin Neuropharmacol. 2002;25(3):158-73.
10. Botte MJ, Abrams RA, Bodine-Fowler SC. Treatment of acquired muscle spasticity using phenol peripheral nerve blocks. Orthopedics. 1995;18(2):151-9.

11. Halpern D, Meelhuysen FE. Phenol motor point block in the management of muscular hypertonia. Arch Phys Med Rehabil. 1966;47(10):659-64.

12. Wood KM. The use of phenol as a neurolytic agent: a review. Pain. 1978;5(3):205-29.

13. Matthews DJ, Balaban B. Management of spasticity in children with cerebral palsy. Acta Orthop Traumatol Turc. 2009;43(2):81-6. DOI: https://doi.org/10.3944/AOTT.2009.081

14. Crema CMT, Santos APBC, Magário APT, Caldas CACT, Riberto M. Neuromuscular block practice in the treatment of spasticity in Brazil. Acta Fisiatr. 2016;23(3):150-4. DOI: https://doi.org/10.5935/0104-7795.20160029

15. Felsenthal G. Felsenthal G. Pharmacology of phenol in peripheral nerve blocks: a review. Arch Phys Med Rehabil. 1974;55(1):13-6.

16. Tilton AH. Management of spasticity in children with cerebral palsy. Semin Pediatr Neurol. 2004;11(1):58-65.

17. Sposito MMM. Bloqueios químicos para o tratamento da espasticidade na paralisia cerebral. Acta Fisiátr. 2010;17(2):68-83. DOI: https://doi.org/10.11606/issn.2317-0190.v17i2a103314

18. Viel E, Pellas F, Ripart J, Pélissier J, Eledjam JJ. Peripheral nerve blocks and spasticity. Why and how should we use regional blocks? Presse Med. 2008;37(12):1793-801. DOI: https://doi.org/10.1016/j.lpm.2008.07.007

19. Kwon JY, Kim JS. Selective blocking of the anterior branch of the obturator nerve in children with cerebral palsy. Am J Phys Med Rehabil. 2009;88(1):7-13. https://doi.org/10.1097/phm.0b013e31815b6381

20. Yadav SL, Singh U, Dureja GP, Singh KK, Chaturvedi S. Phenol block in the management of spastic cerebral palsy. Indian J Pediatr. 1994;61(3):249-55.

21. Elovic EP, Esquenazi A, Alter KE, Lin JL, Alfaro A, Kaelin DL. Chemodenervation and nerve blocks in the diagnosis and management of spasticity and muscle overactivity. PM R. 2009;1(9):842-51. https://doi.org/10.1016/j.pmrj.2009.08.001

22. Brashear A, Lambeth K. Spasticity. Curr Treat Options Neurol. 2009;11(3):153-61.

23. Petrillo CR, Chu DS, Davis SW. Phenol block of the tibial nerve in the hemiplegic patient. Orthopedics. 1980;3(9):871-4.

24. Gracies JM, Elovic E, McGuire JR, Zorowitz R. Traditional pharmacologic treatments for spasticity part i: local treatments. In: Brasher A, Mayer NH. Spasticity: etiology, evaluation, management, and the role of botulinum toxin. New York: Wemove; 2008. p.119-41.

25. Satkunam LE. Rehabilitation medicine: 3. Management of adult spasticity. CMAJ. 2003;169(11):1173-9.

26. O'Brien CF. Treatment of spasticity with botulinum toxin. Clin J Pain. 2002;18(6 Suppl):S182-90.

27. Gormley ME Jr, Krach LE, Piccini L. Spasticity management in the child with spastic quadriplegia. Eur J Neurol. 2001;8 Suppl 5:12735.

28. Karri J, Mas MF, Francisco GE, Li S. Practice patterns for spasticity management with phenol neurolysis. J Rehabil Med. 2017;49(6):482-8. DOI: https://doi.org/10.2340/16501977-2239

29. Deltombe T, De Wispelaere JF, Gustin T, Jamart J, Hanson P. Selective blocks of the motor nerve branches to the soleus and tibialis posterior muscles in the management of the spastic equinovarus foot. Arch Phys Med Rehabil. 2004;85(1):54-8. DOI: https://doi.org/10.1016/S0003-9993(03)00405-2

30. Patel DRL, Soyode O. Pharmacologic interventions for reducing spasticity in cerebral palsy. Indian J Pediatr. 2005;72(10):869-72.

31. Barnes MP. Management of spasticity. Age Ageing. 1998;27(2):239-45. DOI: https://doi.org/10.1093/ageing/27.2.239 
32. Yoshida T, Nakamoto T, Kamibayashi T. Ultrasound-guided obturator nerve block: a focused review on anatomy and updated techniques. Biomed Res Int. 2017;2017:7023750. DOI: https://doi.org/10.1155/2017/7023750

33. Magora F, Rozin R, Ben-Menachem Y, Magora A. Obturator nerve block: an evaluation of technique. Br J Anaesth. 1969;41(8):695-8.

34. Gasparich JP, Mason JT, Berger RE. Use of nerve stimulator for simple and accurate obturator nerve block before transurethral resection. J Urol. 1984;132(2):291-3. DOI: https://doi.org/10.1016/s0022-5347(17)49600-5

35. Choquet $\mathrm{O}$, Capdevila X, Bennourine K, Feugeas JL, BringuierBranchereau S, Manelli JC. A new inguinal approach for the obturator nerve block: anatomical and randomized clinical studies. Anesthesiology. 2005;103(6):1238-45. DOI: https://doi.org/10.1097/00000542-200512000-00020

36. Wassef MR. Interadductor approach to obturator nerve blockade for spastic conditions of adductor thigh muscles. Reg Anesth. 1993;18(1):13-7.

37. Ghai A, Sangwan SS, Hooda S, Garg N, Kundu ZS, Gupta T. Evaluation of interadductor approach in neurolytic blockade of obturator nerve in spastic patients. Saudi J Anaesth. 2013;7(4):420-6. DOI: https://doi.org/10.4103/1658-354x.121074

38. Kakinohana M, Taira Y, Saitoh T, Hasegawa A, Gakiya M, Sugahara K. Interadductor approach to obturator nerve block for transurethral resection procedure: comparison with traditional approach. J Anesth. 2002;16(2):123-6. DOI: https://doi.org/10.1007/s005400200006
39. Jang SH, Ahn SH, Park SM, Kim SH, Lee KH, Lee Zl. Alcohol neurolysis of tibial nerve motor branches to the gastrocnemius muscle to treat ankle spasticity in patients with hemiplegic stroke. Arch Phys Med Rehabil. 2004;85(3):506-8. DOI: https://doi.org/10.1016/S0003-9993(03)00468-4

40. Sook Kim H, Hye Hwang J, Lee PK, Kwon JY, Yeon Oh-Park M, Moon $\mathrm{Kim} \mathrm{J}$, et al. Localization of the motor nerve branches and motor points of the triceps surae muscles in korean cadavers. Am J Phys Med Rehabil. 2002;81(10):765-9. DOI: https://doi.org/10.1097/00002060-200210000-00008

41. Anwar F, Mee H, Ramanathan S. Phenol nerve block for ankle plantar flexor and invertor spasticity in upper motor neuron lesion: a case series. J Int Soc Phys Rehabil Med. 2018;1:55-60.

42. Creze $M$, Peltier J, Havet E, Potier A, Lefranc $M$, Foulon $P$, et al. Anatomy and surgical landmarks for the ansa pectoralis: application to pectoralis major nerve selective neurotomy. Surg Radiol Anat. 2012;34(10):943-51. DOI: https://doi.org/10.1007/s00276-012-0990-3

43. Sefa Özel M, Özel L, Toros SZ, Marur T, Yıldırım Z, Erdoğdu E, et al. Denervation point for neuromuscular blockade on lateral pectoral nerves: a cadaver study. Surg Radiol Anat. 2011;33(2):105-8. DOI: https://doi.org/10.1007/s00276-010-0712-7

44. Kong KH, Chua KS. Neurolysis of the musculocutaneous nerve with alcohol to treat poststroke elbow flexor spasticity. Arch Phys Med Rehabil.1999;80(10):1234-6. DOI: https://doi.org/10.1016/S00039993(99)90021-7 